\title{
Extensive Myocardial Infarction complicated with Stroke as First Presentation of HIV in A young Sudanese Male: Case Report
}

\section{Moh.Mah.Fadelallah Eljack}

University of Bakht Alruda, Faculty of Medicine https://orcid.org/0000-0002-2370-9368

Najla Fouad Nassir Mohammedali

Medani Heart Centre

Omer Idris Ahmed

Medani Heart Centre

Alshareef B. Nour

Wad Madani college of Medical science and technology

Mazin S. Haroun

University of Khartoum, Faculty of Medicine

Khabab Abbasher Hussien Mohamed Ahmed ( $\sim$ Khabab9722@gmail.com )

University of Khartoum, Faculty of Medicine https://orcid.org/0000-0003-4608-5321

Abdallah M. Abdallah

University of Bahri, Faculty of Medicine

Mustafa Mohamed Ibrahim Ali

University of Khartoum, Faculty of Medicine

\section{Case Report}

Keywords: HIV, Myocardial Infarction, Stroke, Sudan

Posted Date: December 1st, 2021

DOI: https://doi.org/10.21203/rs.3.rs-1129448/v1

License: @ (i) This work is licensed under a Creative Commons Attribution 4.0 International License. Read Full License 


\section{Abstract}

HIV can be associated with cardiovascular complications. We highlight unusual presentation of HIV patient with $\mathrm{MI}$ and stroke. 30 year old HIV male presented with MI complicated by stroke. Recovered within 5 days with anti-ischemic and diuretics. Screening tests for unusual presentations prevent fatal complications of common diseases.

\section{Introduction}

A Patient with Human Immunodeficiency Virus (HIV) has an increased risk of developing coronary artery diseases (CAD) either through traditional CAD risk factors, antiretroviral drug effects or HIV-related parameters including inflammatory and immunologic changes (1). Also an increased risk of stroke either including HIV-related intra- and extra-cranial vasculopathy, HIV induced cardiomyopathy, HIV-induced coagulopathy and opportunistic infection-associated vasculitis; However, this is mostly Significant among those younger than 50 , but not among older patients $(2,3)$. The most significant early manifestation of HIV infection among Africans was marked by slim disease (diarrhea and wasting), tuberculosis, variety of Opportunistic Infections (OI), weight loss, fever, and dermatological symptoms(4). Here, we report a young Sudanese male patient whom was diagnosed with Extensive ST segment Elevation Myocardial Infarction (STEMI) complicated later with Ischemic Stroke who was found to have a high HIV viral load with exclusion of other possible causes of his underlying condition.

\section{Case Report}

A 30 years old thin male with a clear medical background presented to the emergency department at Medani Heart Centre (MHC) with central crushing chest pain with maximum intensity radiated to the left shoulder and jaw aggravated by movement and improved by rest not associated with cough or shortness of breath and not improved by ingestion of food or Antacids. The patient denied fever, change in appetite, diarrhea, vomiting, change in urine, headache or any other form of neurological symptoms.

However there was no past history of a similar condition or cardiac problems. The patient mentioned that he is a heavy smoker and has had unprotected sexual intercourse many times. The patient denied drinking alcohol.

On examination the patient looked ill, thin and not pale or jaundiced, pulse 110 regular good volume, Bp100/60 bilaterally, normal pericardium findings, clear chest and soft abdomen. ECG showed Significant ST segment elevation from $\mathrm{v} 1$ to $\mathrm{v} 6$ and mild elevation in leads 2, 3 and avF confirmed later by positive serum troponin as Late Extensive ST segment elevation Myocardial Infarction crushed by aspirin $300 \mathrm{mg}$, clopidogrel $300 \mathrm{mg}$, bisoprolol 2,5 mg, Enoxaparin inj. weight $1 \mathrm{~kg} 12$ hourly and the patient was scheduled for urgent Coronary Angiography but during preparation the ICT for HIV tested positively necessitating confirmation with ELISA which showed a high viral load of $22.4 \mathrm{AU} / \mathrm{ml}$ (normal 
up to $1 \mathrm{AU} / \mathrm{ml}$ ). Other tests revealed normal fasting lipid profile, complete blood count and normal renal function tests with electrolytes.

Echocardiography showed anterior, septal, apical and anterolateral wall hypokinesia with two apical thrombus measures $11 \times 10 \mathrm{~cm}, 10 \times 12 \mathrm{~cm}$ subsequently with moderately impaired left ventricular systolic function (Ejection Fraction=34) favoring the decision of adding furosemide $40 \mathrm{mg}$ tabs once daily and spironolactone tabs $25 \mathrm{mg}$ once daily. See Figure 1

The patient was followed up closely symptomatically with serial ECGs on the subsequent days showing significant improvement until day 4 of Admission in the evening follow up when he was found to have slurred speech and he mentioned that he was unable to move his right side, without mouth deviation, headache or blurring of vision. On examination he was afebrile, conscious, oriented in time, place and person. There was bulbar cranial nerves affection (without fascial palsy), right side hypotonia, power grade 3 and hyporeflexia. The patient was sent for urgent brain CT scan which showed left parietal area infarction. See Figure 2

The patient was followed up for the following 5 days showing complete recovery and returned speech fluency with normal tone, power grade 5 and normal reflexes. He was discharged after being counseled perfectly on full anti-ischemic and diuretics with enoxaparin shifted to rivaroxaban $15 \mathrm{mg} 12$ hourly sent to HIV program for proper management to be seen after two weeks in the Cardiology Referral Clinic.

\section{Discussion}

HIV-infected patients have an increased risk of developing coronary artery disease (CAD), yet short-term prognosis after cardiovascular events remains unclear (5). HIV-related Coronary heart disease risk factors were described in literature, including Antiretroviral therapy, HIV disease parameters, female gender and HCV co-infection (6). CAD etiology in HIV-infected patients is the result of a number of interactions causing inflammation, endothelial dysfunction, and coagulation disorders, ultimately leading to atherosclerosis (7). The severity of HIV-related immunosuppression is correlated with some abnormalities which account for the observed hypercoagulability in HIV-infected patients. This correlation is measured by CD4+ cell counts and the presence of concurrent infectious or neoplastic diseases. These abnormalities include the presence of decreased levels of natural anticoagulants/heparin co-factor dimer, antiphospholipid antibodies/lupus anticoagulant, elevated factor VIII coagulant activity, activated protein $C$ resistance, increased platelet activation and hyperhomocysteinemia $(8,9)$. In our patient, ECG showed Significant ST segment elevation from v1 to $v 6$ and mild elevation in lead 2, 3 and aVF which was confirmed by positive serum troponin as Late Extensive ST segment elevation Myocardial infarction. Myocardial infarction was our initial working hypothesis, yet we included many possible underlying pathologies. At first we excluded HIV opportunistic infections, because myocardial infarction in HIV patients is usually a presentation of the elderly, 50 years of age and more according to studies, unlike our 30 years old previously healthy patient(7). But on our routine out-patient HIV testing program in the emergency department that was done before the urgent coronary Angiography, ICT result for HIV turned 
out positive and was confirmed with ELISA, which showed a high viral load of $22.4 \mathrm{AU} / \mathrm{ml}(10-12)$. After management of myocardial infarction and stabilization of the patient, he suddenly developed stroke. In addition to the traditional risk factors, HIV is associated with other factors that increase the risk of stroke, including immunosuppression and high viremia, as in our patient. The etiology of stroke in people living with HIV is multifactorial, including Atherosclerosis of the large arteries, small vessel disease, cardio embolism, central nervous system (CNS) infections, coagulation disorders, and non-atherosclerotic vasculopathy, yet the triggering mechanism for stroke remains unclear (13). A possible explanation of the presentation at this age is that the myocardial infarction was directly related to HIV chronic inflammation and immune activation independent of other risk factors (6). Two cohort studies found a strong association of HIV with a 44-48\% increased risk of Myocardial Infarction, independent of traditional risk factors, such as age, race, socioeconomic status, and substance abuse $(14,15)$. To our knowledge and available literature, this is the first case ever to present with atypical HIV presentation as an ST-elevation Myocardial Infarction complicated by stroke in such young age.

\section{Conclusion}

This presentation of a young HIV-infected patient is unusual and very uncommon. It points out the risk of subclinical HIV infection, and the increased risk of CAD and stroke in this young HIV-infected population. It also highlights the importance of routine out-patient HIV testing in such emergency cases, in that it reduces the chances of debilitating diseases to go undiagnosed, only to be discovered by its severe complications at a very late age, as in a case of a patient who was diagnosed with HIV for the first time at the age of $74(16)$.

\section{Declarations}

\section{Ethical Approval and Patient's consent:}

Ethical approval was obtained from research and ethics committee at Medani Heart Centre (MHC). Both written and verbal consents were obtained from the patient.

\section{Conflict of interest:}

The Author Report No Conflict of Interest

\section{Funding:}

The Study was funded by authors themselves.

\section{Acknowledgments:}

Not applicable.

\section{Authors' contribution:}


All authors contributed equally in the study.

\section{References}

1. Triant VA. HIV infection and coronary heart disease: An intersection of epidemics. J Infect Dis. 2012;205(SUPPL. 3):355-61.

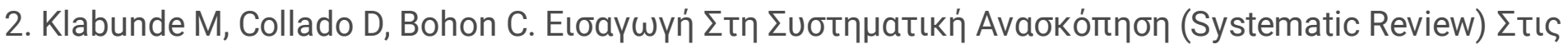

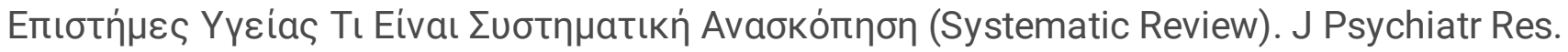
2017;94(3):36-46.

3. Mochan A, Modi M, Modi G. Stroke in black South African HIV-positive patients: a prospective analysis. Stroke. 2003 Jan;34(1):10-5. doi: 10.1161/01.str.0000043821.35051.fa. PMID: 12511743.

4. Tipping $B$, de Villiers $L$, Wainwright $H$, et al. Stroke in patients with human immunodeficiency virus infection. J Neurol Neurosurg Psychiatry. 2007;78(12):1320-1324. [PubMed: 17470469]

5. Okeke NL, Hicks CB, McKellar MS, Fowler VG, Federspiel JJ. History of AIDS in HIV-Infected Patients Is Associated with Higher In-Hospital Mortality Following Admission for Acute Myocardial Infarction and Stroke. J Infect Dis. 2016;213(12):1955-61.

6. Triant VA. Epidemiology of coronary heart disease in patients with human immunodeficiency virus. Rev Cardiovasc Med. 2014;15(SUPPL. 1):1-11.

7. Vachiat A, McCutcheon K, Tsabedze N, Zachariah D, Manga P. HIV and Ischemic Heart Disease. J Am Coll Cardiol. 2017;69(1):73-82.

8. Çilingiroğlu M, Lather N, Youseff A, Helmy T. ST-elevation myocardial infarction due to a spontaneous thrombus in the left anterior descending artery in a young HIV-infected patient. Turk Kardiyol Dern Ars. 2011;39(4):308-11.

9. Ener RA, Palermo J, O’Murchu B, Burke JA, Wolf NM, Van Decker WA. HIV disease in thrombocardiology. J Invasive Cardiol 2007;19:235-9.

10. Hsue PY, Giri K, Erickson S, et al. Clinical fea- tures of acute coronary syndromes in patients with human immunodeficiency virus infection. Circula- tion 2004;109:316-9.

11. Boccara F, Mary-Krause M, Teiger E, et al. Acute coronary syndrome in human immunodefi- ciency virus-infected patients: characteristics and 1 year prognosis. Eur Heart J 2011;32:41-50.

12. Becker AC, Sliwa K, Stewart S, et al. Acute coronary syndromes in treatment-naive black South Africans with human immunodeficiency vi- rus infection. J Interv Cardiol 2010;23:70-7. 56.

13. Bessa PB, Brito AKB, Pereira FR, E Silva SQ, Almeida TVR, Almeida AP de. Ischemic stroke related to HIV and SARS-CoV-2 Co-infection: A case report. Rev Soc Bras Med Trop. 2020;53:1-3.

14. Freiberg MS, Chang CC, Kuller LH, et al. HIV infection and the risk of acute myocardial infarc- tion. JAMA Intern Med 2013;173:614-22.

15. Silverberg MJ, Leyden WA, Xu L, et al. Immu- nodeficiency and risk of myocardial infarction among HIV-positive individuals with access to care. J Acquir Immune Defic Syndr 2014;65:160-6.

16. Gill GS, Govindu RR, Fouda R, Ammar HM. Unexpected Diagnosis. 2019;11(9):1-7. 
Figures

\section{Figure 1}

Echocardiography showed anterior, septal, apical and anterolateral wall hypokinesia

\section{Figure 2}

Brain CT scan which showed left parietal area infarction

\section{Supplementary Files}

This is a list of supplementary files associated with this preprint. Click to download.

- HIVSupp.docx 